\title{
Need of Dark Energy for Dynamical Compactification of Extra Dimensions on the Brane
}

\author{
B. Cuadros-Melgar ${ }^{1,2}$ and E. Papantonopoulos ${ }^{1}$ \\ ${ }^{1}$ National Technical University of Athens, Physics Department, \\ Zografou Campus, GR 157 80, Athens, Greece \\ ${ }^{2}$ Instituto de Física, Universidade de São Paulo, \\ C.P.66.318, CEP 05315-970, São Paulo, Brazil
}

(Received on 5 November, 2005)

\begin{abstract}
We consider a six-dimensional braneworld model and we study the cosmological evolution of a (4+1) braneuniverse. Introducing matter on the brane we show that the scale factor of the physical three-dimensional brane-universe is related to the scale factor of the fourth dimension on the brane, and the suppression of the extra dimension compared to the three dimensions requires the presence of dark energy.
\end{abstract}

\section{INTRODUCTION}

Recent high quality data from various independent observations [1-4] suggest that most of the energy content of our universe is in the form of dark matter and dark energy. Although there have been many plausible explanations for these dark components, it is challenging to try to explain them using alternative gravity theories.

Braneworld models are higher-dimensional modified gravity theories, where matter and gauge interactions are localized on a three-dimensional hypersurface (called brane) in a higher-dimensional spacetime while gravity propagates in all spacetime (called bulk). Contrary to the Kaluza-Klein theories, the extra dimensions can be large if the geometry is non trivial. In a cosmological context however, they are observationally much smaller than the size of our perceived universe. Initially the universe could have started with the sizes of all dimensions at the Planck length and through some mechanism the extra dimensions remained comparatively small during cosmological evolution. Such a mechanism was proposed in [5]. The basic idea is that strings dominate the dynamics of the early universe and can see each other most efficiently in $2(p+1)(p=1$ for strings $)$ dimensions. Therefore, strings can only interact in 3 spatial dimensions, while in higher dimensions they eventually cease to interact efficiently. If branes are included, the mechanism still survives [6].

In this work we show that in a (4+1) braneworld model in a six-dimensional spacetime bulk with a cosmological constant, the unknown form of brane dark energy is responsible for the dynamical compactification of the extra fourth dimension relatively to the physical three dimensions, and during the various cosmological evolution phases of the brane-universe it keeps the extra dimension frozen.

First we consider a 4-brane fixed at some position in the sixth dimension and we derive the dynamical six-dimensional bulk equations in normal gaussian coordinates with a cosmological constant in the bulk and considering matter on the brane [7]. We look for time dependent solutions allowing for two scale factors, the usual scale factor $a(t)$ of the three dimensional space and a scale factor $b(t)$ for the extra fourth dimension. If $a(t)=b(t)$ we get the Friedmann equation of the generalized Randall-Sundrum model in six dimensions describing a four dimensional universe [9]. If $a(t) \neq b(t)$ we get a generalized Friedmann equation in six dimensions [10].

The problem can be looked at a different angle. We consider a 4-brane moving in a static six-dimensional bulk [11, 12]. If $a \neq b$, a brane observer uses $a$ and $b$ to measure the departure from six-dimensional spherical symmetry of the bulk. In order to derive an effective Friedmann equation on the brane [11], $a$ and $b$ are related through the Darmois-Israel junctions conditions and this relation depends on the energymatter content of the brane.

Using the six-dimensional generalized Friedmann equation and assuming differente pressures in the physical three dimensions and in the fourth dimension, we make a systematic numerical study of the cosmological evolution of the scale factors $a(t)$ and $b(t)$ for several values of the parameters of the model. We find that, in order the fourth dimension to be small relatively to the other three dimensions and to remain constant during cosmological evolution, $\hat{w}$ must be negative, indicating the presence of a dark form of energy in the extra fourth dimension.

\section{STATIC BRANE IN A DYNAMICAL BULK}

We consider a six-dimensional spacetime with a metric of the form

$$
\begin{aligned}
d s^{2}=-n^{2}(t, y, z) d t^{2}+a^{2}(t, y, z) d \Sigma_{k}^{2} & +b^{2}(t, y, z) d y^{2} \\
& +d^{2}(t, y, z) d z^{2}
\end{aligned}
$$

where $d \Sigma_{k}^{2}$ represents the 3-dimensional spatial sections metric with $k=-1,0,1$ corresponding to the hyperbolic, flat and elliptic spaces, respectively.

The total energy-momentum tensor can be decomposed in two parts

$$
\tilde{T}_{N}^{M}=\breve{T}_{N}^{M(B)}+T_{N}^{M(b)},
$$

with the energy-momentum tensor on the brane given by

$$
T_{N}^{M(b)}=\frac{\delta\left(z-z_{0}\right)}{d} \operatorname{diag}(-\rho, p, p, p, \hat{p}, 0),
$$

where $\hat{p}$ is the pressure in the extra brane dimension. 
The presence of the brane in $z_{0}$ imposes boundary conditions on the metric. The discontinuity in the metric derivatives generates a delta function in the metric which must be matched with the energy-momentum tensor componentes. Thus, we obtain the following Darmois-Israel conditions

$$
\begin{aligned}
& \frac{\left[\partial_{z} a\right]}{a_{0} d_{0}}=-\frac{\kappa_{(6)}^{2}}{4}(p-\hat{p}+\rho), \\
& \frac{\left[\partial_{z} b\right]}{b_{0} d_{0}}=-\frac{\kappa_{(6)}^{2}}{4}\{\rho-3(p-\hat{p})\} \\
& \frac{\left[\partial_{z} n\right]}{d_{0} n_{0}}=\frac{\kappa_{(6)}^{2}}{4}\{\hat{p}+3(p+\rho)\},
\end{aligned}
$$

where the subscript $(0)$ indicates quantities on the brane. The energy conservation equation on the brane can also be derived from the Einstein equations,

$$
\dot{\rho}+3(p+\rho) \frac{\dot{a}_{0}}{a_{0}}+(\hat{p}+\rho) \frac{\dot{b}_{0}}{b_{0}}=0,
$$

as well as the generalized Friedmann equation in sixdimensions

$$
\begin{array}{r}
\left(\frac{\ddot{a}_{0}}{a_{0}}+\frac{1}{3} \frac{\ddot{b}_{0}}{b_{0}}+\frac{\dot{a}_{0} \dot{b}_{0}}{a_{0} b_{0}}+\frac{\dot{a}_{0}^{2}}{a_{0}^{2}}\right)= \\
-\frac{\kappa_{(6)}^{4}}{32}\left\{\rho\left(\rho+2 p+\frac{2}{3} \hat{p}\right)+(p-\hat{p})^{2}\right\}-2 \frac{k}{a_{0}^{2}}-\frac{\kappa_{(6)}^{2}}{3 d_{0}^{2}} \breve{T}_{66} .
\end{array}
$$

In our case the Einstein equations cannot be integrated analytically and therefore, the usual form of the Friedmann equation on the brane cannot be extracted from (6). We just need a relation between $a$ and $b$ such that (6) will give the cosmological evolution of the scale factor $a(t)$.

$$
\begin{aligned}
& \text { If } a(t)=b(t)=\mathcal{R}(\mathrm{t}) \\
& 2 \frac{\ddot{\mathcal{R}}}{\mathcal{R}}+3\left(\frac{\dot{\mathcal{R}}}{\mathcal{R}}\right)^{2}=-3 \frac{\kappa_{(6)}^{4}}{64} \rho^{2}-\frac{\kappa_{(6)}^{4}}{8} \rho p-3 \frac{k}{\mathcal{R}^{2}}-\frac{\kappa_{(6)}^{2}}{2 d_{0}^{2}} \breve{T}_{6}^{6},
\end{aligned}
$$

which is the generalization of the Randall-Sundrum Friedmann-like equation in six dimensions, and it has, as expected, the $\rho^{2}$ term with a coefficient adjusted to six dimensions.

\section{DYNAMICAL BRANE IN A STATIC BULK}

We consider a 4-brane moving in a six-dimensional static bulk with a metric

$$
d s^{2}=-n(z)^{2} d t^{2}+a^{2}(z) d \Sigma_{3}^{2}+b(z)^{2} d y^{2}+d^{2}(z) d z^{2} .
$$

Introducing an energy-momentum tensor on the brane

$$
\hat{T}_{\mu v}=h_{v \alpha} T_{\mu}^{\alpha}-\frac{1}{4} T h_{\mu \nu}
$$

where $T_{\mu}^{\alpha}=\operatorname{diag}(-\rho, p, p, p, \hat{p})$, the Darmois-Israel conditions

$$
\left[K_{\mu v}\right]=-\kappa_{(6)}^{2} \hat{T}_{\mu \nu}
$$

give the equations of motion of the brane

$$
\begin{aligned}
\frac{d^{2} \dot{d} \dot{\mathcal{R}}^{3}-d \ddot{\mathcal{R}}}{\sqrt{1+d^{2} \dot{\mathcal{R}}^{2}}}-\frac{\sqrt{1+d^{2} \dot{\mathcal{R}}^{2}}}{n} \times & \left.-\left(d \partial_{z} n-n \partial_{z} d\right) \dot{\mathcal{R}}^{2}\right) \\
\times\left(d \dot{n} \dot{\mathcal{R}}+\frac{\partial_{z} n}{d}\right. & =-\frac{\kappa_{(6)}^{2}}{8}(3(\rho+p)+\hat{p}), \\
\frac{\partial_{z} a}{a d} \sqrt{1+d^{2} \dot{\mathcal{R}}^{2}} & =-\frac{\kappa_{(6)}^{2}}{8}(\rho+p-\hat{p}), \\
\frac{\partial_{z} b}{b d} \sqrt{1+d^{2} \dot{\mathcal{R}}^{2}} & =-\frac{\kappa_{(6)}^{2}}{8}(\rho-3(p-\hat{p})) .
\end{aligned}
$$

Equation (11) is the main dynamical equation describing the movement of the brane-universe in the six-dimensional bulk, while a combination of (12) and (13) acts as a constraint relating $a$ and $b$

$$
a=\mathcal{A} b^{(\rho+p-\hat{p}) /(\rho-3(p-\hat{p}))} .
$$

This is the main result of our paper: because of the DarmoisIsrael conditions, the relative cosmological evolution of $a$ and $b$, depend on the dynamics of the energy-matter content of the brane-universe. Another interesting observation is that, a brane observer can measure the departure from full sixdimensional spherical symmetry of the bulk using the quantities $a$ and $b$.

\section{THE COSMOLOGICAL EVOLUTION OF THE BRANE-UNIVERSE}

We assume that the universe started as a four-dimensional one at the Planck scale with all the dimensions at the Planck length and matter isotropically distributed. Then an anisotropy was developed in the sense that $\hat{p}=Q p$ with $Q \neq 1$. The matter distribution on the brane is given by

$$
\begin{aligned}
& p=w \rho, \\
& \hat{p}=\hat{w} \rho .
\end{aligned}
$$

From the generalized Friedmann equation the cosmological evolution of the three-dimensional scale factor $a(t)$ is given by

$$
\begin{array}{r}
{\left[1+\frac{B}{3}\right] \ddot{a} a^{2 C+1}+\left[\frac{B^{2}}{3}+\frac{2 B}{3}+1\right] \dot{a}^{2} a^{2 C}} \\
+\frac{\kappa_{(6)}^{2}}{32} a^{2}\left[1+2 w+\frac{2}{3} \hat{w}+(w-\hat{w})^{2}\right] \\
-a^{2 C}\left[a^{2} \frac{\kappa_{(6)}^{2}}{3} \Lambda_{6}-2 k\right]=0
\end{array}
$$

where

$$
\begin{aligned}
& B=\frac{1-3 w+3 \hat{w}}{1+w-\hat{w}}, \\
& C=3(1+w)+B(\hat{w}+1),
\end{aligned}
$$


and

$$
b(t)=a(t)^{B} .
$$

We will make a numerical analysis of equations (17) and (20) and study the time evolution of the two scale factors for different backgrounds and spatial brane-curvature. For the scale factor $b(t)$ to be small compared to the scale factor $a(t)$, the constant $B$ in (20) should be negative. In Table 1 we give the allowed range of values of $\hat{w}$ for various values of $w$. The criterion for the acceptance of a solution is to give a growing evolution of $a(t)$ and a decaying and freezing out evolution for $b(t)$.

\begin{tabular}{|c|lll|}
\hline$w$ & \multicolumn{3}{|c|}{$\hat{w}$} \\
\hline-1 & $>0$ & or & $<-4 / 3$ \\
\hline 0 & $>1$ & or & $<-1 / 3$ \\
\hline $1 / 3$ & $>4 / 3$ & or & $<0$ \\
\hline$-1 / 3$ & $>2 / 3$ & or & $<-2 / 3$ \\
\hline
\end{tabular}

TABLE I: The allowed values of $\hat{w}$ for $B$ to be negative.

\section{A. De Sitter Bulk}

For $\Lambda_{6}>0$ only negative values of $\hat{w}$ give acceptable solutions for $k=0,-1$, while the $k=1$ solutions are not acceptable for any value of $\hat{w}$.

As we can see from Fig. 1 and 2, the scale factor $a(t)$ grows very fast, while the scale factor of the fourth dimension goes very fast to small values where it stays constant for the whole evolution. The reason for the fast growth of $a(t)$ is that the cosmological constant of the bulk $\Lambda_{6}$ acts as an effective cosmological constant on the brane and drives an exponential growth. This is common to the braneworld models with a cosmological constant in the bulk. It also happens in the fivedimensional Randall-Sundrum model if we do not impose the fine tuning between the brane tension and the five-dimensional cosmological constant.

\section{B. Anti De Sitter Bulk}

For $\Lambda_{6}<0$ and $k=-1$ there is an interesting evolution of an oscillating universe shown in Fig. 3, again only for negative values of $\hat{w}$. For $w<-1$ there is a small range of values near the critical value of $\hat{w}$, where $a(t)$ escapes from the oscillating behaviour and grows very fast, forcing $b(t)$ to decay and freeze out at certain small value analogously to De Sitter cases.

\section{Minkowski Bulk}

For $\Lambda_{6}=0$ we do not have the very strong effect of the bulk cosmological constant. We have physical solutions for $k=0,-1$ as we can see in Fig. 4. The evolution of $a(t)$

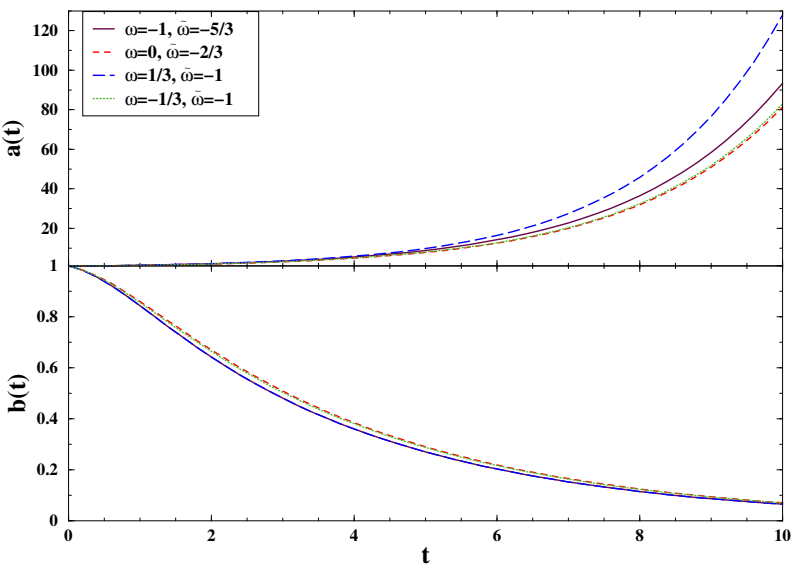

FIG. 1: Time evolution of the scale factors $a(t)$ and $b(t)$ for $\Lambda_{6}>0$ and $k=0$.

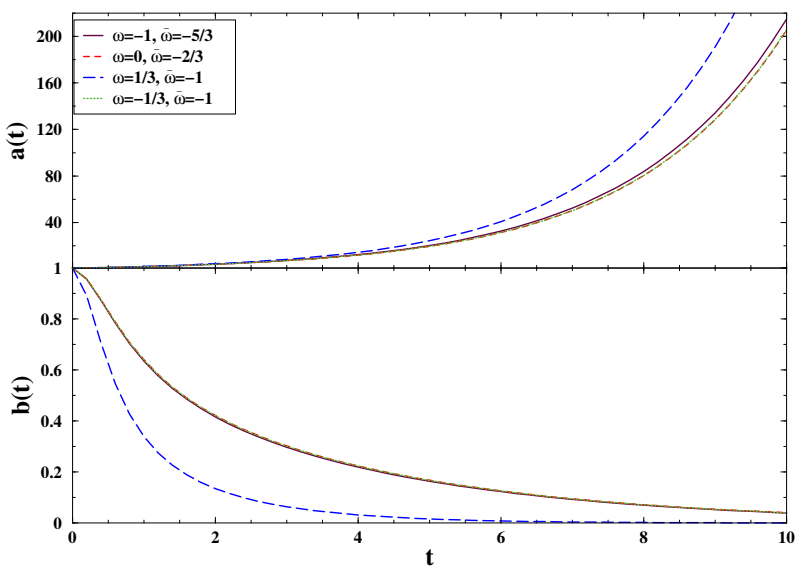

FIG. 2: Time evolution of the scale factors $a(t)$ and $b(t)$ for $\Lambda_{6}>0$ and $k=-1$.

is nearly linear for $k=-1$, while for $k=0$ there is an interesting solution with dark energy in the physical universe $(w=-1)$ and with "phantom" energy in the extra dimension $(\hat{w}=-5 / 3)$. The $b(t)$ scale factor decays at a smaller rate compared with the case of a non-zero bulk cosmological constant. As it happens in all the other cases, $\hat{w}$ is negative in the range of values given in Table 1 for all acceptable solutions, indicating the need of dark energy to suppress the extra fourth dimension compared to the three other dimensions.

\section{CONCLUSIONS}

We presented a (4+1)-braneworld cosmological model in a six-dimensional bulk where the four-dimensional universe 


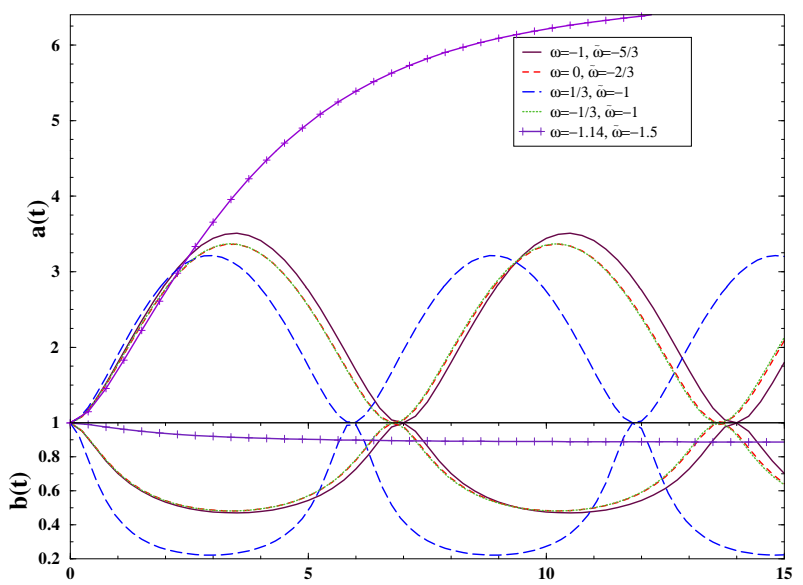

FIG. 3: Time evolution of the scale factors $a(t)$ and $b(t)$ in an oscillating universe for $\Lambda_{6}<0$ and $k=-1$.

evolves with two scale factors. Demanding an effective Friedmann-like equation on the brane the motion of the 4brane in the static bulk is constrained by Darmois-Israel conditions. Thus, $a$ and $b$ are related in a way that depends on the energy-matter content of the 4-brane.

We assumed that the universe started higher-dimensional at the Planck scale with all the dimensions at the Planck length, and subsequently an anisotropy was developed between the three physical dimensions and the extra-dimension. In all considered cases we found that dark energy is needed for the dy- namical suppression and subsequent freezing out of the extra fourth dimension.

Acknowledgement: We would like to thank E. Abdalla, A.

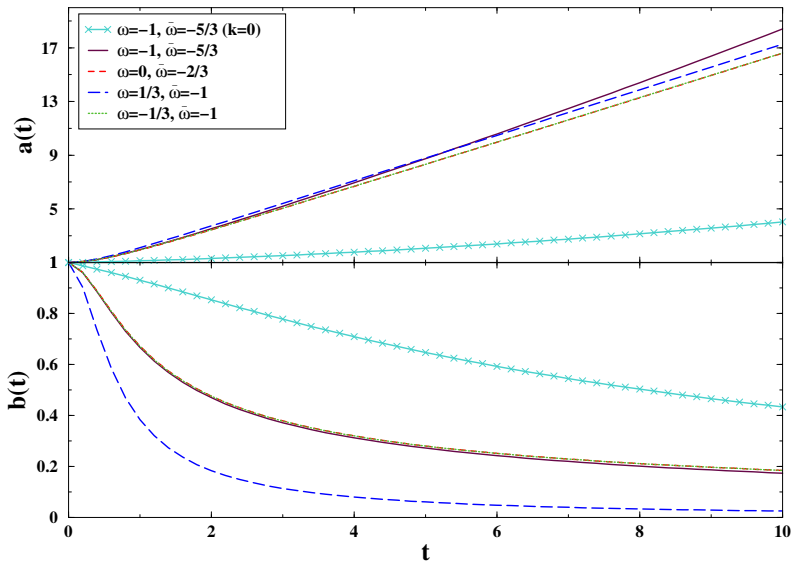

FIG. 4: Time evolution of the scale factors $a(t)$ and $b(t)$ for $\Lambda_{6}=0$ and $k=0,-1$.

Kehagias and R. Maartens for useful discussions and BCM would like to thank NTUA for its hospitality. This work was partially supported by the Greek Education Ministry research program "Pythagoras" and by Fundação de Amparo à Pesquisa do Estado de São Paulo (FAPESP), Brazil.
[1] D. N. Spergel et al. [WMAP Collaboration], Astrophys. J. Suppl. 148, 175 (2003).

[2] R. Scranton et al. [SDSS Collaboration], [arXiv:astro$\mathrm{ph} / 0307335]$

[3] A. G. Riess, [arXiv:astro-ph/9908237].

[4] G. Goldhaber et al. [The Supernova Cosmology Project Collaboration], [arXiv:astro-ph/0104382].

[5] R. H. Brandenberger and C. Vafa, Nucl. Phys. B 316, 391 (1989).

[6] S. Alexander, R. H. Brandenberger and D. Easson, Phys. Rev. D 62, 103509 (2000).

[7] P. Binetruy, C. Deffayet, U. Ellwanger and D. Langlois, Phys. Lett. B 477, 285 (2000).
[8] P. Binetruy, C. Deffayet and D. Langlois, Nucl. Phys. B 565, 269 (2000)

[9] E. Abdalla, A. Casali and B. Cuadros-Melgar, Nucl. Phys. B 644, 201 (2002) [arXiv:hep-th/0205203].

[10] B. Cuadros-Melgar, "Gravitational Shortcuts in Brane Cosmology", PhD Thesis, Examined June 2003, Universidade de São Paulo, Brazil.

[11] C. Csaki, J. Erlich and C. Grojean, Nucl. Phys. B 604, 312 (2001). P. Bowcock, C. Charmousis and R. Gregory, Class. Quant. Grav. 17, 4745 (2000).

[12] B. Cuadros-Melgar, Class. Quant. Grav. 21, 2669 (2004). 